\title{
Hacia una nueva propuesta teórica de sistematización de los estratos del texto narrativo. Fábula, trama y relato como planos funcional, actuacional y discursivo
}

\section{Towards a New Theoretical Proposal of Systematizing of the Levels in the Narrative Text. Story, Plot and Narrative as Levels of Functions, Actions and Discourse}

\author{
José R. Valles Calatrava \\ Universidad de Almería \\ jrvalles@ual.es
}

\section{RESUMEN}

Este artículo repasa en primer término las propuestas teóricas sobre los estratos del texto narrativo para posteriormente realizar una nueva propuesta que diferencia tres planos (fábula/ funciones, trama/actuaciones, relato/discurso) que asimismo se conectan con las instancias enunciativas (autor/lector empírico, autor/lector implícito, narrador/narratario) y los cuatro elementos fundamentales (personajes, acción, espacio, tiempo) del texto narrativo.

Palabras Clave: Texto narrativo; Fábula; Historia; Trama; Intriga; Relato; Discurso.

\section{ABSTRACT}

This article examines in the first term the theoretical proposals about the narrative levels. Later, it realizes a new proposal that differentiates three levels (story/function, plot/action, narrative/discourse) that it joins in the same way with the instances of the enunciation (empirical author/reader, implied author/reader, narrator/narratee) and the four basic elements of the narrative text (characters, action, space, time).

Key words: Narrative Text; Story; Plot; Narrative; Discourse.

\section{BREVE PANORAMA DE LAS PRINCIPALES PROPUESTAS TEÓRICAS ${ }^{1}$ SOBRE EL TEMA}

La cuestión de los estratos del texto narrativo, entendiendo por tales los distintos planos de ordenación, configuración y presentación del material die-

\footnotetext{
${ }^{1}$ Pueden verse unas versiones mucho más breves e incompletas de este primer epígrafe en dos de mis libros (Valles, 2002: 354-358 y 2008: 84-89).

Copyright: (C) 2016 CSIC. Este es un artículo de acceso abierto distribuido bajo los términos de una licencia de uso y distribución Creative Commons Attribution (CC-by) España 3.0.
} 
gético en el mismo, es sin duda una de las más abordadas por la teoría de la narrativa y, también sin duda, una de las menos convenidas entre los distintos estudios y estudiosos. Al siempre espinoso tema de las traducciones de ciertos términos a otras lenguas, se unen una gran variedad de denominaciones ${ }^{2}$ y un desacuerdo conceptual sobre el alcance de los distintos estratos, también inestables en lo tocante a su propio número, que — como puede verse en el cuadro que cierra este primer epígrafe - hacen de esta distinción, en realidad una abstracción teórica con finalidad esencialmente metodológica ${ }^{3}$, una de las menos estables de la narratología.

Si bien el asunto de la ordenación lógica del material y su organización literaria ya estaba planteada en la teoría aristotélica de la fábula $-\mu \hat{v} \theta o s-$, en la diferenciación clásica entre ordo naturalis y ordo poeticus y, en cierto modo, en la propia oposición retórica entre la inventio y la dispositio, es a los formalistas rusos a quienes cabe el mérito de ser los primeros en retomar en el siglo XX esa distinción, sin duda una de las de mayor éxito y continuidad - y asimismo más debatidas — de la trayectoria histórica de la narratología. Tal oposición metodológica, pues en el texto literario tales planos son realmente inseparables y simultáneos, existiendo físicamente solo la — digamos- materialidad discursiva usualmente verbal y gráfica, separa la fábula, el contenido que se narra en el texto, y la trama — sjužet, literalmente suje$t o^{4}$, originariamente en ruso сюжет- o disposición literaria real, efectiva y concreta de los materiales de tal fábula en tal texto.

La primera noción, pues, en tanto que relación lógico-temporal de los acontecimientos o motivos, marca la virtualidad de la narración y su representación y sumarización mental, correspondiendo al plano profundo o, si se quiere, al ordo naturalis y la inventio retórica; la segunda, como ordenación literaria efectiva de los mismos, supone la actualización de la primera como realidad discursiva concreta y se relaciona con el ordo poeticus y la dispositio, y en cuanto a su diseño para la ejecución verbal efectiva con la elocutio; ambas, por tanto, se encuentran en distinto orden en las trayectorias de escritura (historia-trama) y lectura (trama-historia) (Garrido Domínguez, 1993: 39; Segre, 1976: 34-35). Escribe Tomashevski:

${ }^{2}$ Eludo voluntariamente el término nivel, reservándolo para la posición de la instancia narrativa respecto al relato según la idea de Genette (1972: 238-241) y para no aumentar aún más la variedad terminológica.

${ }^{3}$ Según Reis y Lopes, «[...] esta dicotomía debe ser considerada como mero instrumento operatorio susceptible de elucidar algunos aspectos esenciales de la composición macroestructural de cualquier texto narrativo y no como distinción absoluta de dos dominios autónomos, existentes $a$ se» (Reis-Lopes, 1995: 118). Habría que añadir que la oposición podría funcionar también para sustentar las cuestiones de adaptación y transducción (Doležel, 1986) o transmedialidad, pero también para explicar las organizaciones macroestructurales de otros relatos, sean estos narrativos y artísticos (cine, novela gráfica), no artísticos (información, historia, relato natural) o incluso, en la dirección dramatológica de García Barrientos (1991), dramáticos (comedia, sainete), y hasta representativos, artísticos y no verbales (ballet, performance). 


\begin{abstract}
Llamamos trama [fábula] al conjunto de acontecimientos vinculados entre sí que nos son comunicados a lo largo de la obra. La trama podría exponerse de una manera pragmática, siguiendo el orden natural, o sea el orden cronológico y causal de los acontecimientos, independientemente del modo en que han sido dispuestos e introducidos en la obra. La trama se opone al argumento [trama, sjužet], el cual, aunque está constituido por los mismos acontecimientos, respeta en cambio su orden de aparición en la obra y la secuencia de las informaciones que nos lo presentan. En una palabra: la trama es lo que ha ocurrido efectivamente; el argumento es el modo en que el lector se ha enterado de lo sucedido (Tomashevski, 1982: 202; ver también Sullà, 1996: 40-55).
\end{abstract}

Aunque tanto Tomashevski como Shklovski comparten los mismos criterios generales de esta distinción, hay sin embargo diferencias, pues para el último el sjužet no es solo la ordenación artística de la materia por narrar sino, asimismo y como precisa Erlich (1974), la totalidad de los recursos utilizados en el relato concreto de la fábula. Además, si los procedimientos y técnicas narrativas expuestas por Shklovski se centraban en la organización del sjužet, los análisis de Tomashevski de los motivos y, ulteriormente, los de Propp de las funciones se ligan más bien al estudio de la fábula. En su Teoría de la prosa Shklovski afirma:

El concepto de trama [sjužet $\left.{ }^{5}\right]$ se confunde muy a menudo con la descripción de los acontecimientos, con aquello que propongo llamar convencionalmente fábula. En realidad, la fábula no es otra cosa que el material para realizar la trama. De esta manera la trama de Evgeni Onegin no es la relación amorosa entre Onegin y Tatiana, sino la elaboración formal de esta fábula mediante la inserción de digresiones que la interrumpen (Shklovski, 1975: 178).

La distinción entre plot/story se debe a Edward M. Forster, a partir de una de sus conferencias dictadas en el Trinity College y luego publicadas conjuntamente en su muy revisitado por la crítica inglesa (Bourneuf y Ouellet, 1985: 45) volumen Aspectos de la novela. Aunque ciertamente ambos conceptos se vinculan y corresponden de modo general con el de fábula/trama de los formalistas rusos o el de historia/discurso de los estructuralistas, se oponen fundamentalmente por la ordenación temporal/causal de los acontecimientos y, además, tienen un origen más intuitivo y una formulación menos precisa y elaborada. Para Forster la story sería la secuencia de los hechos temporalmente ordenados; provocarían en el lector el deseo de saber lo que va a suceder, interrogándose a sí mismo con preguntas como «¿y después?», «¿y luego?» El plot sería, en cambio, la estructura narrativa de los hechos desde una perspec-

${ }^{4}$ Rodríguez Pequeño (1995: 170) da cuenta de la cercanía del sentido del término al usado por los comparatistas para aludir al tema; se aproximaba así al inglés subject o al francés sujet.

${ }^{5}$ Son patentes no solo las diferencias sino las contradicciones terminológicas entre las traducciones de los textos de Shklovski, que empleó antes la distinción, y Tomashevski, quien la fijó poco después más concretamente. 
tiva causal, la configuración lógica de la historia en la novela; el plot provocaría un efecto estético mediante las técnicas narrativas de montaje y composición y generaría asimismo la participación de la memoria y la inteligencia del lector. Aclara el novelista y teórico inglés:

Definamos el argumento [plot]. Hemos descrito la historia [story] como una narración de sucesos ordenada temporalmente. Un argumento es también una narración de sucesos, pero el énfasis recae en la causalidad. Una historia es: «El rey murió y luego murió la reina». Un argumento es: «El rey murió y luego murió la reina de pena». Se conserva el orden temporal, pero se ve eclipsado por la sensación de causalidad (Forster, 1983: 92).

No obstante, si bien la oposición story/plot de Forster ha tenido mucha acogida en el ámbito anglosajón, también en ocasiones se ha modernizado y reorientado, abierto y precisado, el segundo par. Jeremy Hawthorn, por ejemplo, deja de lado el orden causal marcado por Forster para considerar que «a plot is an ordered, organized sequence of events and actions» (Hawthorn, 1997: 125), y en este mismo sentido coinciden tanto el Diccionario de Cuddon ( $«$ the plan, design, scheme or pattern of events in a play, poem or work of fiction») como la Nueva Enciclopedia de Princeton («plot may be defined as the pattern or structure of events within a text») (Cuddon, 1998: 676; Preminger y Brogan, 1993: 916). Peter Brooks entiende que plot «is the principle of interconnectedness and intention which we cannot do without moving the discret elements —incidents, episodes, actions — of a narrative» (Brooks, 1984: 13). Y Paul Ricoeur, en su trabajo «Narrative Time» (1980: 169-190), considerando la estrecha relación existente entre el tiempo, la narrativa y la historicidad, acentúa la dimensión temporal del plot: mientras «the story is a collection of stand-alone events» y tiene por tanto una dimensión episódica, el plot, «the intelligible whole that governs a succession of events in any story», acentúa no solo la relación temporal entre los acontecimientos sino que muestra además un tiempo interior de cada uno de ellos además de una conexión concreta con su historicidad.

Partiendo del concepto de motivo de Tomashevski pero sobre todo del de motivema de Alan Dundes que replantea el anterior, Doležel elaboró en «From Motifemes to Motifs» (1972: 55-90) un modelo articulado de funcionamiento de los motivemas y motivos en relación a los estratos textuales narrativos: 1) en el estrato de la fábula y ordenados causal y sucesivamente, se encuentran los motivemas, proposiciones generales y abstractas que predican un acto vinculado a un actante; 2) en el plano de la intriga y en una ordenación literaria, se hallan los motivos, realizaciones textualmente variables que materializan el acto como acción y el actante como personaje; 3) en el nivel de la textura, con variaciones narrativas y estilísticas, se encuentran los enunciados narrativos que verbalizan discursivamente los motivos de la intriga. Bastante posteriormente, Chico Rico acomodó esta división a los planteamientos de la lingüís- 
tica textual denominando respectivamente macrocomponente semántico, macrocomponente sintáctico y microestructura narrativa a estos planos:

El orden secuencial lógico y cronológico de los motivemas, producto de la operación retórica de inventio, constituirá el macrocomponente semántico, fábula del texto narrativo; el orden secuencial particular de los motivos, consecuencia de la acción de la dispositio sobre el anterior nivel, será la base del macrocomponente sintáctico, sujeto o intriga del texto narrativo; en fin, el tejido actualizará el último nivel como microestructura narrativa, discurso merced a la elocutio retórica (Chico Rico, 1988: 82).

En la narratología estructuralista tuvo especial importancia la división historia/discurso, que recogía la distinción de los formalistas rusos entre fábula y sujeto, entre ordenación lógica causotemporal de los acontecimientos y su disposición literaria concreta. Ya el propio Benveniste, considerando algunas ideas de Buyssens, distinguía en sus Problemas de lingüística general (1971) entre dos sistemas distintos y complementarios, el de la historia y el del discurso, a partir del uso de los tiempos verbales que tienen referencia al momento del suceso (indefinido) o al del habla (presente, pretérito perfecto) y de otras formas (pronombres personales de primera y segunda persona, deícticos) que también aluden sólo al tiempo del discurso, y, sobre todo, por la necesaria presencia de una voz narrativa en el campo del discurso (Culler, 1978). Tzvetan Todorov distingue asimismo entre historia y discurso, entre lo que se cuenta y cómo se cuenta, y los relaciona respectivamente con la oposición formalista entre fábula y sujeto y la retórica de inventio y dispositio: la obra literaria es historia en la medida en que representa una realidad en la que ocurren unos acontecimientos e intervienen unos personajes, y es discurso al ser referida la historia por un narrador que la organiza de una determinada manera. Escribe Todorov:

Es historia en el sentido de que evoca una cierta realidad, acontecimientos que habrían sucedido, personajes que, desde este punto de vista, se confunden con los de la vida real. (...) Pero la obra es al mismo tiempo discurso: existe un narrador que relata la historia y frente a él un lector que la recibe. A este nivel, no son los acontecimientos referidos los que cuentan, sino el modo en que el narrador nos los hace conocer (Todorov, 1974: 157).

La historia, además, recogería los planos semánticos — lo que representa el relato- y sintáctico — relaciones entre las unidades narrativas- de la obra; el discurso, en cambio, se conectaría con el plano verbal — propiedades estilísticas y punto de vista-. El conocido caso de los Ejercicios de estilo de Raymond Quenau, donde una misma historia se cuenta de 99 formas diferentes, ilustra perfectamente esta distinción narratológica.

Por su parte, ya anteriormente, en su trabajo de 1966 para el mítico número 8 de Communications, Roland Barthes (1974: 15-38) se dejaba llevar de otros criterios para diferenciar tres - y no dos- niveles en el texto narrati- 
vo: el de las funciones, el de las acciones y el de la narración. Domínguez Caparrós (2002: 166) ha sistematizado las similitudes y diferencias existentes entre la propuesta de Barthes y las posteriors de Todorov y Segre — que más adelante examinaré- en el siguiente cuadro:

$\begin{array}{lll}\text { TODOROV } & \text { BARTHES } & \text { SEGRE } \\ \text { Relato como historia } & \text { Nivel de las funciones } & \text { Fábula } \\ & \text { Nivel de las acciones } & \text { Intriga } \\ \text { Relato como discurso } & \text { Nivel de la narración } & \text { Discurso }\end{array}$

La división historia/discurso ha tenido numerosas aplicaciones y ha sido objeto de distintos replanteamientos. Fuera del ámbito francés, Seymour Chatman, en su conocido libro Story and Discourse. Narrative Structure in fiction and Film (1990) o, mucho más recientemente y en España, Darío Villanueva (1989: 15-17), mantienen la distinción de dos estratos en el texto narrativo, el qué o historia y el cómo o discurso, correspondientes, según el último (1989: 15-17), a los respectivos planos del contenido y la forma, algo que de modo muy parecido recoge Bremond (1973) al separar lo relatado y relatante, el récit racconté y el récit raccontant.

Y asimismo una oposición conceptual y terminológicamente muy similar es la de Gerald Prince entre narrated/narrating, lo narrado y lo narrante, si bien esta, aparte de ser equivalente en una primera acepción a la distinción entre la ordenación causal y cronológica frente a la literaria (historia/discurso), también ofrece un segundo sentido específico contraponiendo lo enunciado y el acto enunciativo. Estas son las dos entradas de A Dictionary of $\mathrm{Na}$ rratology (1987):

Narrated. 1. The set of situations and events recounted in a narrative; the STORY (as opposed to the DISCOURSE). 2. The signs in a narrative representing the situations and events narrated (as opposed to the NARRATING).

[...] Narrating. 1. The telling or relating of one or more events. 2. The DISCOURSE (as opposed to the STORY). 3. The signs in a narrative representing the narrating activity, its origin, its destination, and its context (as opposed to the NARRATED) (Prince, 1987: 56 y 57).

Previamente, en 1979, en Theorie des Erzählens, el austriaco Franz Stanzel revisó algunos de sus planteamientos a la luz de otras investigaciones narratológicas y, partiendo de su categoría de mediación entre «la historia y cómo es contada esta», reflejó en el llamado círculo tipológico (Stanzel, 1988: XI y XVI) — que no marca fronteras estrictas sino límites graduales de transicióntres situaciones narrativas y tres diferentes categorías en oposiciones binarias que se combinan en tal círculo. Las categorías son las de modo (narrador/reflector), persona (identidad/no identidad entre narrador y personaje ficticio) y perspectiva (perspectivismo/aperspectivismo o interna/externa) y las tres situaciones narrativas, en las que prevalece siempre como dominante una categoría, son: en primera persona, en que el narrador pertenece al mundo ficticio 
y prima la identidad del mundo de narrador y personaje; auctorial, en que el narrador no pertenece al mundo ficticio y prima la perspectiva externa; figural, en que el narrador es sustituido por un reflector, o elemento dominante que siente y observa pero no cuenta (Stanzel, 1988; Gnutzmann, 1994: $207-$ 213).

En Figures III (1972), Genette replanteará las oposiciones binarias formuladas por los formalistas, Benveniste o Todorov separando la segunda — sjužet o discurso- en dos niveles y proponiendo distinguir así tres planos distintos dentro del discurso narrativo: la historia o contenido que se cuenta, el relato o manifestación discursiva - ya textual y concreta- del mismo y la narración o acción de narrar, que por tanto incluiría a las otras dos (Genette, 1972; Scholes, 1981). La historia, pues, es expuesta en un relato concreto a partir de la enunciación narrativa; según el teórico francés:

Je propose [...] de nommer histoire le signifié ou contenu narratif [...], récit proprement dit le signifiant, énoncé, discours ou texte narratif lui-même, et narration l'acte narratif producteur et, par extensión, l'ensemble de la situation réelle ou fictive dans laquelle il prend place ${ }^{6}$ (Genette, 1972: 72).

Y, veinte años después, en Nouveau discours du récit (1998), insiste en los mismos términos y conceptos, defiende su relativa diferencia y sobre todo pertinencia de la triada frente a las parejas formalista y estructuralista, aunque rectifica su orden, $\mathrm{y}$, haciendo depender tanto al relato como a la historia de la narración, precisa la jerarquía y adscripción disciplinar de los mismos:

El verdadero orden, en un relato no ficticio (histórico, por ejemplo) es, por supuesto, historia (los acontecimientos desarrollados), narración (el acto narrativo del historiador) y relato, el producto de esa acción, susceptible, en teoría o en la práctica, de sobrevivir como texto escrito, grabación, recuerdo humano. [...] el relato designa el discurso pronunciado (aspecto sintáctico y semántico, de acuerdo con los términos de Morris) y la narración, la situación en la que se profiere: aspecto pragmático (Genette, 1998: 13).

Si la propuesta de Rimmon-Kenan en Narrative Fiction: Contemporary Poetics (1983) mantiene conceptualmente la distinción ternaria de Genette, tan solo sustituyendo uno de los términos (historia/texto/narración), otra perspectiva distinta es la de Greimas y Courtès que en Semiótica. Diccionario... (1991) delimitan el objeto y niveles procedimentales del análisis semiótico, distinguiendo en el texto el nivel de manifestación o del lenguaje-objeto (el conjunto significante que aparece en el texto y se analiza) y el nivel descriptivo o de la gramática narrativa (elementos del metalenguaje semiótico utilizado en la descripción); este último estaría integrado por dos subniveles: el nivel de superficie o discursivo, a su vez conformado por el componente narrativo (en-

\footnotetext{
${ }^{6}$ Me disculpo de antemano por tener que mantener la cita en francés, al no tener a mano la traducción española del conocido libro de Genette.
} 
cadenamiento de estados y transformaciones) y el componente discursivo (encadenamiento de figuras y efectos de sentido), y el nivel profundo o semiótico, integrado por un primer y un segundo plano de organización.

En cambio, Mieke Bal presenta en Narratology (1985) un esquema ternario como Genette, pero muy distinto: la fábula es la organización causocronológica de motivos, la historia es la presentación de la anterior de un determinado modo literario, mediante los aspectos, y, finalmente, el texto narrativo es el discurso que, sobre las dimensiones anteriores, narra un agente. Escribe concretamente Bal:

Un texto narrativo será aquel en que un agente relate una narración. Una historia es una fábula presentada de cierta manera. Una fábula es una serie de acontecimientos lógica y cronológicamente relacionados que unos actores causan o experimentan.

La fábula, entendida como material al que da forma la historia, se ha definido como una serie de acontecimientos. Dicha serie se construye siguiendo ciertas leyes. Llamamos a esto la lógica de los acontecimientos. [...] Los acontecimientos, los actores, el tiempo y el lugar constituyen conjuntamente el material de una fábula. [...] Los elementos se organizan de cierta manera en una historia [...] El resultado de estos múltiples procesos es una historia específica, distinta de otras historias. Me referiré a los rasgos específicos de una historia dada como aspectos.

[...] Una fábula que se ha ordenado en una historia no es todavía un texto. Un texto narrativo es una historia que se cuenta con un lenguaje, esto es, que se convierte en signos lingüísticos (Bal, 1985: 13-15).

Onega y García Landa siguen los planteamientos de Bal ejemplificando su tripartición en Robinson Crusoe:

[...] We will say that the text is the linguistic artifact that we can buy and read, written de facto by Defoe and supposedly by Robinson. The fabula is whatever happened to Robinson in his travels and on his island. The story is the precise way in which that action is conveyed, the way the fabula is arranged into a specific cognitive structure of information (Onega y García Landa, 1996: 6).

El propio García Landa (1990 —edición online de 2005-) realizó previamente el siguiente cuadro para representar el que llama esquema vertical de Bal:

Author

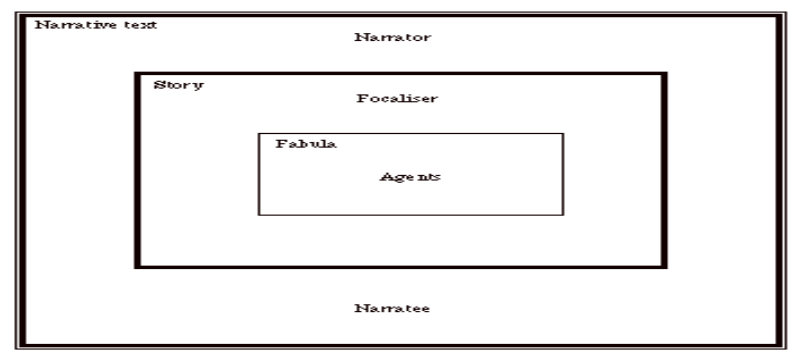

Reader 
Finalmente, los semióticos Cesare Segre y Carmen Bobes presentan unas parecidas propuestas organizativas de los niveles narrativos textuales.

Segre (1976 1985), que entiende que la cronología y la causalidad estructuran la intriga y alteran la fábula, relacionando el texto narrativo y sus estratos con el contexto cultural y los papeles del emisor y el receptor, distingue el modelo narrativo o forma más general de exposición de un relato sin alterar sus conexiones, la fábula — «el contenido, o mejor sus elementos esenciales, colocado en un orden lógico y cronológico»—, la intriga — «el contenido del texto en el mismo orden en que se presenta»- y el discurso — «el texto narrativo significante»- (Segre, 1976: 14). En uno de los modelos más completos, el semiótico italiano relaciona respectivamente tales planos con cuatro niveles culturales (conceptos clave y lógica de la acción, materiales antropológicos, técnicas expositivas y lengua) que funcionarían también como vínculos del texto con el contexto en el que actúan emisor y receptor.

Bobes (1993) en cambio diferencia en un sentido puramente metodológico la historia - totalidad de motivos-, el argumento — modo y orden de presentación literaria de los mismos- y el discurso - dimensión verbosimbólica-:

\footnotetext{
Manifestados sincréticamente en el discurso novelesco único y total pueden distinguirse metodológicamente tres aspectos, sin que suponga reconocer partes objetivamente separables, distinguibles en una forma discreta: la historia, o conjunto de motivos, el argumento, o forma y orden en que se exponen los motivos, y el discurso, o signos lingüísticos que los expresan [...] En el esquema que proponemos, el discurso es lo más inmediato, lo que se nos ofrece en la lectura. En él desglosamos teóricamente, liberándolo de sus palabras textuales, el argumento, y ordenando los motivos en la línea temporal que tendrían fuera del texto, llegamos a la historia (Bobes, 1993: 50-51 y 141).
}

Si intentamos sistematizar las distintas propuestas de estratos narrativos diferenciadas por los más importantes teóricos, se podría elaborar el siguiente cuadro (1), que amplía por nuestra parte en varias filas de teóricos [Shklovski y Tomashevski, Forster, Doležel y Chico Rico, Benveniste, Todorov y Villanueva, Bremond, Rimmon-Kenan, Onega-García Landa, Segre y Bobes] el realizado por Fludernik (1996: 62) y recogido por Hawthorn (2000: 336): 
CUADRO 1

SISTEMATIZACIÓN DE LAS APORTACIONES TEÓRICAS

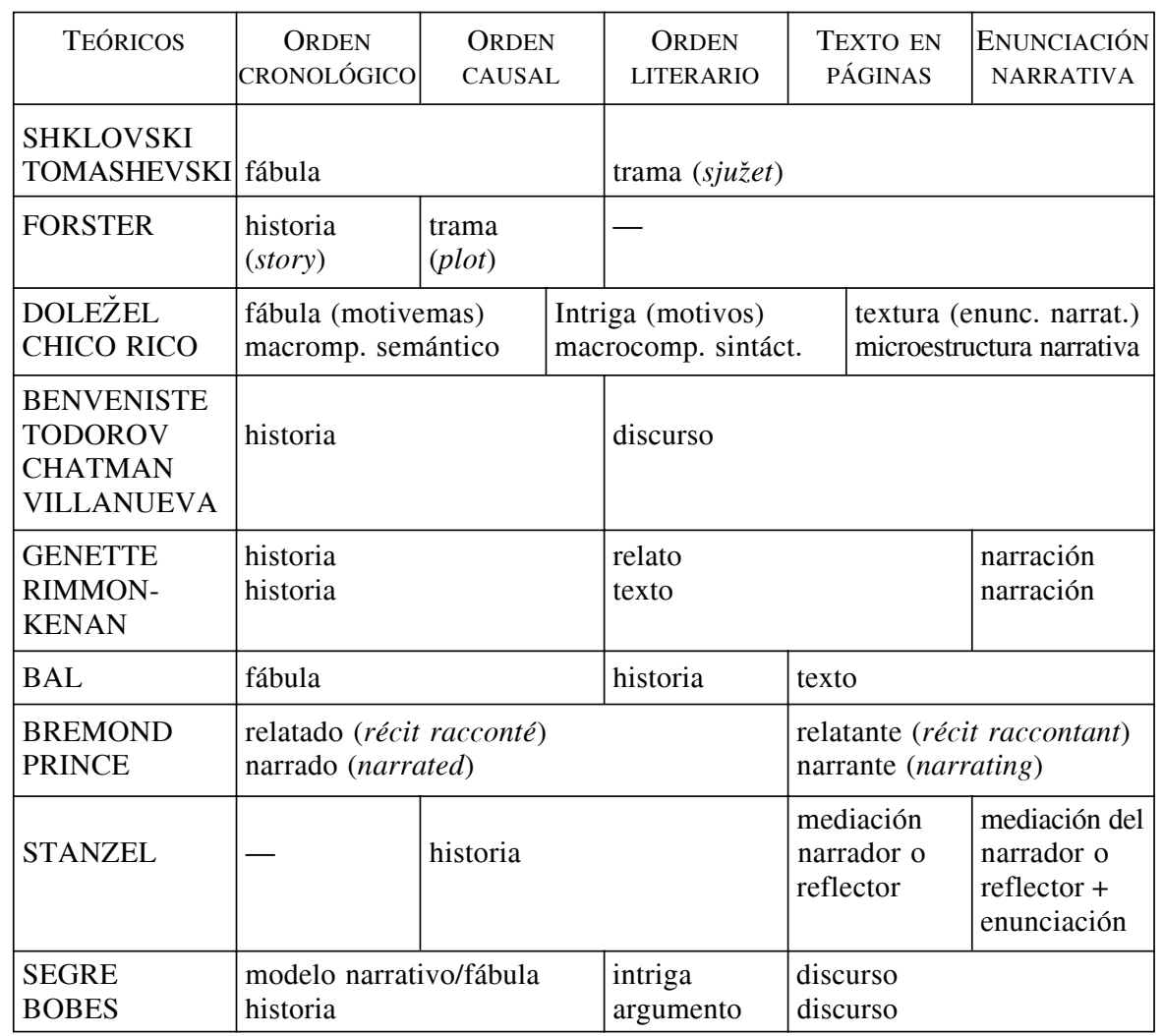

\section{HACIA UNA NUEVA PROPUESTA DE SISTEMATIZACIÓN DE LOS ESTRATOS DEL} TEXTO NARRATIVO Y SU RELACIÓN CON OTROS ELEMENTOS DEL MISMO

Como se ha podido apreciar, en una propuesta de abstracción teórica con fines básicamente metodológicos — como es esta de los estratos o planos narrativos-, la dispersión, e incluso confusión, no solo terminológica sino conceptual es más que notable ${ }^{7}$. A mi entender, esto se debe en alguna medida a problemas de traducción y de diferencias lingüísticas, pero se debe también, y sobre todo, a la diversidad de los modelos teóricos subyacentes a las pers-

\footnotetext{
${ }^{7}$ El propio Genette (1998: 12-13) certifica, aludiendo a su distinción previa sobre este tema en Figures II y a la lejanía y/o impropiedad de ciertas divisiones binarias previas, que «es muy fácil perderse».
} 
pectivas individuales que estudian el texto narrativo, e incluso a la falta de deslinde de las distintas clases y órdenes de cuestiones teóricas que se concitan bajo cada conceptualización. Creo, pues, que esta debe ser la primera misión y a ella responde el primer epígrafe.

Pero pienso asimismo que los estratos narrativos se deben vincular igualmente con otros elementos del texto narrativo (personajes, acción, espacio, tiempo) y con otras instancias de la enunciación (autor-lector —empíricos o implícitos-, narrador-narratario), a pesar de que ello, como se ha visto, haya contribuido a generar también parte de la confusión. A intentar elaborar unas propuestas teóricas y metodológicas coherentes de tales correlaciones se dedican los dos siguientes y respectivos epígrafes.

\subsection{Fábula, trama y relato como estratos del texto narrativo}

En primer lugar, parece aceptada en casi todas las distinciones modernas la organización trimembre de estratos narrativos. Salvo las antiguas distinciones de los formalistas (fábula/sjužet), Forster (story/plot) y los estructuralistas (historia/discurso), además de Bremond y Prince (récit racconté/récit raccontant, narrated/narrating), el resto de modelos teóricos de abstracción, más recientes, optan por una tripartición de planos conceptuales (Genette, Bal, Doležel, Segre, Bobes, etc.) en los que uno de los elementos fijos es el estrato de los discursos efectiva y verbalmente emitidos por el narrador (o narradores y personajes), existiendo sin embargo más vacilaciones en cuanto a los otros dos conceptos de la terna.

Sucede eso, a mi entender, porque bajo tales conceptos subyacen tres clases de problemas teóricos que habría que deslindar: el primero afecta al objeto del estrato, el segundo a su naturaleza y el tercero al orden de sus elementos. En cuanto al objeto que cubre cada estrato, propongo diferenciar el relato o discurso narrativo, lo relatado o contenido narrado y la lógica del relato, o macroestructura narrativa según van Dijk (1972, 1980); sobre el carácter o naturaleza del plano, cabría distinguir entre palabras, actuaciones y funciones (dimensiones verbal/escénica/funcional); con respecto al orden, pueden oponerse el textual (tipográfico), el literario o propiamente narrativo y el cronológico y causal. Los respectivos estratos que los cubrirían, en correspondencia con algunas de las ideas de Segre y con los tres niveles propuestos en su momento por Barthes (de la narración, de las acciones y de las funciones), serían el relato, la trama y la fábula: el primer plano es enunciativo y está definido por los signos verbosimbólicos; el segundo es el propiamente narrativo, referido a las acciones y actuaciones contadas por el narrador vinculándolas a los personajes; el tercero es el macroestructural y lógico, que está vinculado a las categorizaciones y representaciones conceptuales. 
Mi selección y elección de los mencionados términos pretenden atenerse en lo posible tanto a su propiedad teórica como a su tradición de uso especializado y sentidos en español ${ }^{8}$. Relato, en referencia al discurso narrativo (como asimismo solo discurso) me parece más que apropiado, fijado y comúnmente aceptado tras su reorientación semántica a ese sentido especializado en teoría de la narrativa por Genette. Trama, mejor que intriga o argumento, pues, si bien ambas denominaciones se han empleado frecuentemente en nuestro ámbito cultural dentro de la historia de esta cuestión, la primera tiene una acepción en el DRAE muy coincidente con nuestra idea de su significado, mientras que intriga no ofrece ningún uso literario en nuestro sentido y argumento alude a cuestiones temáticas y más cercanas casi a la fábula. He dudado mucho más respecto a historia y fábula, porque, siendo ambos teóricamente idóneos, acaso sea algo más amplio el bagaje de uso especializado del primer término, si bien el $D R A E$ recoge una acepción bastante cercana a nuestro concepto en fábula, y prácticamente no ofrece un sentido literario especializado en historia ${ }^{9}$.

Tendríamos pues un texto narrativo que, pese a su indivisibilidad objetiva y a su materialidad verbal y (usualmente) tipográfica, dividimos, en un proceso de doble abstracción teórica, en tres estratos metodológicamente operativos, que guardan asimismo correlación con otros elementos e instancias enunciativas del citado texto:

- El primero, el más material, es el relato, un plano enunciativo constituido por las palabras, que atiende al discurso narrativo (el relato), muestra una dimensión verbosimbólica y dispone sus elementos en un orden textual.

- El segundo y superior, el plano de las acciones contadas por el narrador, sería la trama, que se refiere al contenido narrativo (lo relatado) y se ofrece en una dimensión escénica que conecta distintas actuaciones y que las organiza, según el orden dado por el narrador, en una disposición literaria.

- Por fin, el tercero y último, la fábula, es un estrato lógico que se muestra como una superestructura narrativa del relato ordenando, causal y cronológicamente y en una dimensión funcional, las categorizaciones y representaciones lógicas de distintos elementos del texto narrativo (funciones/motivos o su enlace en secuencias).

${ }^{8}$ Por su tradición y aceptación, he optado por mantener en inglés (en la traducción del título de este artículo) story y plot, añadiendo narrative (en coherencia con el uso de Chatman y la traducción al inglés de la distinción de Genette) por relato.

${ }^{9} D R A E$ : Trama. 4. Disposición interna, contextura, ligazón entre las partes de un asunto u otra cosa, y en especial el enredo de una obra dramática o novelesca. Argumento. 2. Asunto o materia de que se trata en una obra. Fábula 3. En las obras de ficción, trama argumental. Historia. 7. Narración inventada. 
Pueden sistematizarse tales planos y características en el cuadro (2) que a continuación se incluye:

CUADRO 2

ESTRATOS DEL TEXTO NARRATIVO

\begin{tabular}{|c|c|}
\hline ESTRATOS NARRATIVOS & OBJETO, CARÁCTER Y ORDENACIÓN \\
\hline $\begin{array}{c}\text { RELATO } \\
\text { plano enunciativo: } \text { PALABRAS }\end{array}$ & $\begin{array}{l}\text { - El relato (discurso) } \\
\text { - Dimensión verbal (palabras) } \\
\text { - Orden textual }\end{array}$ \\
\hline $\begin{array}{c}\text { TRAMA } \\
\text { plano narrativo: ACTUACIONES }\end{array}$ & $\begin{array}{l}\text { - Lo relatado (contenido) } \\
\text { - Dimensión escénica (actuaciones) } \\
\text { - Orden literario }\end{array}$ \\
\hline $\begin{array}{c}\text { FÁBULA } \\
\text { plano lógico: CATEGORIZACIONES }\end{array}$ & $\begin{array}{l}\text { - Lógica del relato (macroestructura) } \\
\text { - Dimensión funcional (funciones) } \\
\text { - Orden cronológico y causal }\end{array}$ \\
\hline
\end{tabular}

\subsection{Fábula, trama y relato en su relación con las instancias enunciativas}

Cabe, en un paso más, plantear la vinculación de los mencionados estratos narrativos con las instancias enunciativas, tanto extra como intratextuales, en concreto los dobletes autor-lector (empíricos) de un lado y autor-lector (implícitos) y narrador-narratario de otro ${ }^{10}$.

Intratextualmente, narrador y narratario (y en su caso distintos personajes que asuman estos roles) se muestran como las instancias emisora y receptora del discurso narrativo, el relato, por lo que se correlacionan particularmente con este último; entretanto, stricto sensu la narración y la audición son, en sentido genettiano (Genette, 1998: 13), actos de enunciación y recepción que incluyen, en su seno y a partir de la materialidad verbal del primero, a los tres inseparables estratos narrativos (relato, trama, fábula), pero además la enunciación narrativa y, según el mismo teórico (Genette, 1972), las transformaciones temporales (orden, duración, frecuencia) y de modo (distancia, focalización) y voz (narrador-narratario, persona, instancia, tiempo y niveles de la narración) coadyuvan a la constitución del relato en relación a la trama. En cambio, la pareja del autor y el lector implícito son transposición a categorías intratextuales de sus correlatos empíricos y se sitúan dentro del texto pero fuera del discurso del narrador (y de los personajes narradores).

En La casa de Asterión de El Aleph de Borges (1980: 52-54), el paratexto titular, la cita inicial, la dedicatoria final, los términos entre corchetes, las frases

\footnotetext{
${ }^{10}$ Eludo voluntariamente aquí, al no ser el objeto final de este trabajo, el ingente y casi inacabable número de citas y referencias vinculadas a cualquiera de los elementos —con ese $\mathrm{u}$ otros nombres similares - de estos tres dobletes.
} 
en cursiva y la nota a pie de página [de un supuesto traductor, en realidad un autor implícito mostrado como fautor (Tacca, 1978: 34-63)] son marcas intratextuales pero extranarradoras del autor implícito [o implicado, implied autor (Booth, 1974)] que contribuyen a completar-elucidar el sentido interpretativo final que puede desprenderse de la estrategia de lectura demandada intratextualmente al lector implícito (Iser, 1976): una forma de [primera] lectura crecientemente sorprendente en sentido prospectivo ante el aumento del suspense [¿quién es Asterión?, ¿de qué casa me hablan?] y un replanteamiento mental posterior y reconstructivo, elucidatorio y resituador, de los signos indiciales manifiestos y preguntas implícitas existentes en el texto [ahora entiendo quién es Asterión, claro que esa era su casa...]. Pero también son engañosas las instancias enunciativas responsables de la narración y audición del relato, el narrador y el narratario: no son el narrador-personaje que habla, medita y recuerda, sobre sí y su casa, tan extraña y detenidamente, ante alguien indeterminado, durante casi todo el cuento, sino un narrador heterodiegético y una focalización omnisciente, que han permitido el uso de un soliloquio o monólogo citado (Cohn, 1981: 11-17) de un personaje, para aparecer solo al final y — con dos frases directas, la mención de dos personajes y la reproducción de las palabras de uno de ellos, dirigidas asimismo a un narratario indeterminado e innominado - dejar clara la dependencia del relato de su posición narrativa y revelar anticlimáticamente la solución del enigma de la identidad del personaje Asterión y de su rara vivienda.

En lo relativo a las instancias extratextuales, tales estratos, fábula, trama y relato, se ofrecen aquí en respectiva correspondencia con las partes retóricas de la inventio, la dispositio y la elocutio, en una asociación ya examinada y realizada por distintos teóricos, para correlacionarlos con tres aspectos sucesivos de la dimensión pragmática de la comunicación literaria. Con respecto al autor empírico extratextual, los tres pares, fábula-inventio, trama-dispositio y relato-elocutio, podrían ser conectados con las tres fases creativas: ideación, organización y escritura; del otro lado, en un proceso inverso y en cuanto a la recepción lectora real y efectiva, empírica, se corresponderían con los procesos semasiológicos de la lectura, la comprensión y la interpretación.

Este ámbito de correlación de las actividades autorales y lectorales con los estratos textuales, particularmente con el de la superestructura lógica del mismo, la fábula, ha sido especialmente desarrollado por los estudios de psicología cognitivista aplicados a la literatura en general y a la narrativa muy en particular que exploran la relación de la misma con los universales cognitivos y la organización general del pensamiento. Núñez Ramos (2010) y Garrido Domínguez (2011) han sintetizado en España algunas de las principales aportaciones al estudio del relato generados desde esta perspectiva ${ }^{11}$.

${ }^{11}$ Cabe citar, colateral y muy brevemente aquí, por su evidente conexión con los temas que estoy abordando, algunos trabajos de narratología cognitivista. En su trabajo sobre los 
Siguiendo con el mismo ejemplo, Borges idea una tematización del conocido mito del Minotauro y el laberinto de Creta pero invirtiendo el mismo: jugando con el conocimiento general pero muy sumario de la historia mítica, dispone el cuento invirtiendo la perspectiva de los hechos. Para ello, deposita visión y voz durante la mayoría del breve relato en un Asterión que, desde su ajenidad respecto al mundo e ignorancia de su destino, va cifrando y graduando las informaciones que permiten adivinar su identidad y su vivienda; finalmente,

universales narrativos, Sternberg (2003a y b) considera que la narrativa no vive en o sobre el tiempo sino entre tiempos y que somos los lectores/oyentes los que los construimos; así, por ejemplo, considera universales del relato al suspense, la curiosidad y la sorpresa, que respectivamente definen las dinámicas lectoras de la prospección, la retrospección y el reconocimiento y que constituyen tres distintas operaciones funcionales generales de la mente en su forma de organizar temporalmente los diferentes modos expositivos narrativos (Sternberg, 2003a: 326 y ss.). Pero, además de su propio estudio, en los dos volúmenes publicados en Poetics Today, Sternberg establece una importante recapitulación de los planteamientos y consideraciones cognitivistas aplicados al relato - algunos de ellos ya citados-, desde los trabajos iniciales de, por ejemplo, George Lakoff y Mark Johnson o Andrew Ortony hasta los numerosos estudios más recientes de William F. Brewer, David Bordwell, Monika Fludernik, Richard Gerrig y David Allbritton, Luc Herman, Manfred Jahn, Paul E. Jose, E.W. Kneepens, David E. Rummelhart, Marie-Laure Ryan o Rolf A. Zwaan: la teoría de la narrativa cognitivista ha desarrollado así cuestiones como la estructura general del relato y del pensamiento narrativo frente al lógico, la metáfora y el relato, la dilación, el suspense y la expectativa psicológica en el mismo, la emoción y la estructura del relato, los fenómenos de apertura y cierre (comienzo/conclusión) y la organización semántica del mismo, el modelo de comprensión literaria, la construcción del personaje, los topoi psicoculturales y su aparición en los relatos, las estrategias de generación de empatía y afecto del lector en el relato, la elaboración psíquica de los mundos posibles (Sternberg, 2003a y b; Valles, 2008: 254), etc. En fin, todo un abanico de amplios temas que afectan al funcionamiento del relato como estructura mental universal y como medio de relación, cognición y representación del mundo.

Mencionaré asimismo a L. Zunshine, quien sostiene en Why We Read Fiction: Theory of Mind and the Novel (2006) cómo nuestra mente conjetura y confirma determinadas hipótesis sobre la actuación y rasgos de los personajes y cómo mediante la metarrepresentación somos capaces de atribuir los discursos a los personajes responsables originalmente de los mismos, esto es, atribuir la responsabilidad discursiva a las distintas, cambiantes y jerarquizadas instancias enunciativas, así como algunos artículos contenidos en el volumen editado por Herman (2003): «Cognitive Maps and the Construction of Narrative Space» de M.-L. Ryan sostiene la existencia de un modelo mental de representación espacial que permite ir situando distintos acontecimientos, evocaciones o palabras dentro del espacio narrativo; «Natural Narratology and Cognitive Parameters» de M. Fludernik parte de la capacidad omniexplicativa de la narratología universal y examina cómo cuatro determinados parámetros cognitivos permiten interpretar los relatos por los lectores y catalogar los mismos en niveles narrativos, desde los esquemas básicos de comprensión de la acción hasta los más elevados de adscripción de macrogénero, pasando por los intermedios de lectura perspectivista del material (Acción, Narración, Experiencia, Puntos de Vista, Reflexión) y de los marcos architextuales o de género; «Constructing Social Space: Sociocognitive Factors in the Interpretation of Character Relations» de C. Emmott interpreta a la luz del cognitivismo los dobletes de correlaciones enunciativas autor-lector, autor implícitolector implícito, narrador-narratario y personaje-personaje (Herman, 2003: 214-242, 243-267 y 295-321; Garrido Domínguez, 2011: 168-174). 
aclarando el enigma y cerrando el suspense, el texto hace emerger a un narrador y personajes cuyos meros nombres ya revelan la conexión del sorprendente relato en perspectiva invertida con nuestro conocimiento del mismo mito, pero con otra fábula, otra trama y por supuesto otras enunciaciones narrativas distintas a estas — como siempre - fascinantes y subyugantes, raras y misteriosas, palabras de Borges. Y todo lector, cualquier lector, aparte de sus propias particularidades personales del proceso lectoral e interpretativo, va quedando intrigado y prendado del texto en la primera lectura, sorprendido y admirado tras la comprensión de la anagnóris del mismo, deudor de la magia verbal y la circularidad narrativa de Borges al correlacionar este relato mítico nuevo y distinto con un conocido mito de siempre.

La citada relación de los estratos textuales con las instancias enunciativas intra y extratextuales puede representarse en el siguiente cuadro (3):

CUADRO 3

ESTRATOS TEXTUALES E INSTANCIAS ENUNCIATIVAS

\begin{tabular}{|c|c|c|c|c|c|}
\hline AUTOR & & & Texto & & LECTOR \\
\hline $\begin{array}{c}\text { Ideación } \\
\downarrow\end{array}$ & $\begin{array}{l}\text { Autor } \\
\text { implícito }\end{array}$ & $\begin{array}{l}\mathrm{N} \\
\mathrm{A} \\
\mathrm{R}\end{array}$ & $\begin{array}{l}\text { FÁBULA } \\
\text { (inventio) }\end{array}$ & $\begin{array}{lc}\text { A } & \text { Lector } \\
\text { U } & \text { implícito } \\
\text { D } & \end{array}$ & $\begin{array}{c}\text { Interpretación } \\
\uparrow\end{array}$ \\
\hline $\begin{array}{c}\text { Organización } \\
\downarrow\end{array}$ & & $\begin{array}{l}\mathrm{A} \\
\mathrm{C}\end{array}$ & $\begin{array}{c}\text { TRAMA } \\
\text { (dispositio) }\end{array}$ & $\begin{array}{l}\mathrm{C} \\
\mathrm{C}\end{array}$ & $\begin{array}{c}\text { Comprensión } \\
\uparrow\end{array}$ \\
\hline Escritura & Narrador & Ó & $\begin{array}{l}\text { RELATO } \\
\text { (elocutio) }\end{array}$ & $\begin{array}{l}\text { Ó } \\
\text { N Narratario }\end{array}$ & Lectura \\
\hline
\end{tabular}

\subsection{Fábula, trama y relato en su relación con los elementos narrativos}

Como en el caso de las instancias enunciativas, los estratos textuales guardan relación asimismo con los distintos componentes comúnmente destacados en el texto narrativo, esto es, con los personajes, acción, tiempo y espacio.

En realidad, dado que lo que objetivamente existen son las estructuras verbosimbólicas textuales, todos esos elementos están presentados y dibujados, ordenados y configurados, verbalmente, y ello tanto en una dirección sintagmática como paradigmática, horizontal como vertical: personajes, acción, espacio y tiempo son el resultado de la aplicación de nuestras operaciones cognitivas a la configuración discursiva de y en cada uno de estos cuatro ámbitos, son la integración de la verbalidad y las operaciones narrativas del texto con 
la cognición del sujeto. Así pues y por lo mismo, plantear ahora en este nuevo sentido que un personaje (1) es, también e inclusivamente, un actor (2) y un sujeto actancial, héroe o protagonista (3) supone aceptar, en coherencia con esos tres estratos textuales señalados, que podemos abstraerlo y definirlo teóricamente en otros planos y que podemos diferenciar, consecuentemente con esos estratos, distintas facetas o papeles del mismo, proyectándolas metodológicamente a plataformas de análisis narrativos diferentes aunque interconectados.

Propongo por ello diferenciar tres dimensiones en cada uno de estos elementos narrativos (personajes, acción, tiempo, espacio) que conforman los mundos ficcionales literarios en coherencia con los tres estratos narrativos señalados (relato, trama, fábula) ${ }^{12}$.

En el plano discursivo del relato, se mantienen las denominaciones de personajes, acción, tiempo y espacio para referirse a estos ámbitos construidos mediante la interacción de la cognición con la verbalidad y las operaciones narrativas (temporalización, modo, voz, trandiscursivización, y transtextualidad, técnicas narrativas y composición textual, etc.) y que incluyen a las otras dos facetas o dimensiones de los mismos. Todos los elementos aparecen discursivamente configurados: denominación, rasgos, identidad, cosificación o transfiguración, caracterización directa o diseminada, transfiguraciones, dobles y tipos, retratos o esperpentizaciones, etc. en los personajes; precisiones o imprecisiones temporales, acronías y ucronías, ralentizaciones y escenas, roturas de orden, duración y frecuencia cronológicas, etc. en el tiempo del relato; acciones simples y complejas, contrarias, dobles o subordinadas, matizadas o esquemáticas, paralelismos e inversiones o flip-flops, encadenamientos, enclaves y circularidades, estructuras en díptico, tríptico, políptico o caleidoscopio, etc. en la acción; descripción, hiperdescripción y cosismo, formas espaciales y desespacialización, atmósferas simbólicas, lugares personalizados, etc. en el espacio.

En el nivel inmediatamente superior de la trama, encontraríamos, en una dimensión actuacional y un orden literario dado por el narrador, solo facetas de los mismos en relación a la actuación, casi como si se tratara de una escena teatral: los personajes son solo actores, las acciones acontecimientos, tiempo y espacio se fusionan para crear un ámbito de actuación extensivo y durativo (Valles, 1999: 139-141), que acoge el proceso de realización de un suceso. El núcleo de esta dimensión escénica es el acontecimiento, porque su ejecución

${ }^{12}$ En realidad, como han hecho casi todos los teóricos, al enlazar con estos distintos estratos los distintos elementos del texto narrativo, si bien con distintas denominaciones y distribuciones a partir de las diferentes posiciones teóricas y visiones personales. Pero con una diferencia: yo planteo claramente que se trata de un proceso de abstracción teórica para distinguir, no cuestiones distintas, sino tres facetas textuales de cada uno de estos cuatro elementos narrativos. 
procesual, su desarrollo de principio a fin, marca la duración y extensión tempoespacial y el cambio y apertura hacia otro acontecimiento nuevo y distinto, como si de una (serie de) escena(s) teatral(es) o narrativa(s) se tratase; el personaje aparece como actor agente o paciente de los acontecimientos, quedando definido por tanto, aparte de esto, de un lado por la perspectiva de la posición y la permanencia o del movimiento y el itinerario, de otro por la actuación, sea esta dinámica, gestual, verbal o interior (Valles, 2002: 499-503); el ámbito de actuación constituye la fusión durativa y extensional del tiempo y el espacio, que se ofrece como un desde dónde y cuándo y un hasta dónde y cuándo, acogiendo el proceso del acontecimiento.

En el tercer estrato de la fábula, el de la macroestructura lógica ${ }^{13}$, estos elementos quedan reordenados al ser dispuestos causal y cronológicamente y

${ }^{13}$ La organización lógica del relato, que se sitúa en la misma base del planteamiento sobre las secuencias de Bremond (1973 y 1974) y luego Todorov (1973 y 1974), fue ya planteada por Aristóteles con respecto a la tragedia. La estructura tripartita de tal secuencia [simple] en el relato, integrada por una exposición, un desarrollo y un desenlace, se vincula también con las nociones de prótasis, epítasis y catástrofe como procesos respectivos y sucesivos de planteamiento del problema, intensificación y evolución hasta la crisis del mismo y resolución o finalización de este. La denominada pirámide de Freytag, expuesta en 1894 y referida asimismo a la estructura del drama, es en su organización diagramática igualmente aplicable a la composición de la fábula de cualquier relato: en dicha figura geométrica integra y representa una doble lógica de la acción: de un lado, los factores propios de la intensidad o tensión dramática o narrativa (momento incitante, acción en ascenso, clímax, acción en descenso, momento de último suspense); de otro, los componentes de la estructura del argumento (exposición, complicación, inversión, catástrofe). Apoyándose en ellos, otros teóricos han incidido asimismo en su organización lógico-estructural, estableciendo esquemas quinarios de la articulación de la acción narrativa. Así, como recoge Garrido (1993: 56-57), Larivaille distingue lo que precede (estado inicial) o continúa (estado final) al comienzo o conclusión respectiva de los acontecimientos, mediando entre ambos un proceso de transformaciones en tres etapas consecutivas (Provocación-Acción-Sanción); Isenberg se apoya en la sociolingüística y la psicolíngüística americana para establecer otro esquema (Orientación-Complicación-Evaluación-ResoluciónMoral-eja-) en el que sucesivamente se ordenan la presentación de constituyentes del relato, aparición del conflicto, reflexión de los protagonistas, final del proceso narrativo y lección o consecuencias. Para la lingüística del texto de van Dijk (1974-1975, 1980), frente a las estructuras lingüísticas textuales (macro y microestructura) existen otras no lingüísticas, sobre todo la superestructura, una especie de organización semántica inter y sobretextual, de esquema general categórico, jerárquico y reglado del diseño textual: las dos superestructuras fundamentales son, para van Dijk, la narrativa y la argumentativa, que permanecen inmutables pese a las respectivas variaciones de las historias y las premisas o conclusiones; un texto narrativo debería obedecer al siguiente modelo canónico organizativo, de composición triádica básica aunque expandible a una fórmula quinaria: exposición-complicación-resolución-(evaluación)(moral). Todos estos modelos sobre la organización lógica de la acción coinciden en articularse respecto a los conceptos de comienzo (principio, inicio, apertura), medio (nudo, complicación) y conclusión (fin, desenlace, meta), no solo como partes textuales de la superestructura lógica del relato sino asimismo como verdaderos núcleos constructores, regidores y organizadores del desarrollo de la acción (Valles, 2008: 159).

Con la estructura de la lógica narrativa se relacionan, evidentemente y asimismo, otros conceptos vinculados al ámbito de la fábula o superestructura lógica de lo relatado, que son 
recategorizados al ser traspuestos a una dimensión funcional: como detenidamente ha estudiado la narratología — sobre todo la estructural—, los personajes son actantes o figuras (o esferas de acción, héroes, antagonistas, figurantes, personajes primarios, etc.), los acontecimientos son motivos o funciones (delito, búsqueda, interrogatorio, etc.; o secuencias — simples o complejas-, si se conectan lógicamente estas), tiempo y espacio se transforman respectivamente en momentos y lugares como marcos que acogen actantes y funciones, como meros receptáculos o emplazamientos, bien topográficos bien cronológicos.

Utilizaré de nuevo el cuento de La casa de Asterión de Borges, una breve narración de personaje y espacio ya desde el paratexto titular, para resumir, muy brevemente, estas facetas triangulares de los elementos narrativos en los tres distintos planos de la fábula (3), la trama (2) y el relato (1).

Con respecto al personaje, Asterión es: (3) un sujeto-protagonista; (2) el actor que medita sobre sí y su casa, sale a la calle, asusta y huye, reflexiona sobre su unicidad, corre por el laberinto, juega y conversa con un amigo imaginario, mata sin quererlo a los humanos, se interroga sobre su destino; (1) un personaje, con ascendencia, nombre e identidad, reconocible finalmente como un minotauro, autorretratado y autocaracterizado indicial y diseminadamente con diversos rasgos (solitario, ingenuo, inculto, extraño, humano, bondadoso aunque aterrorizador, etc.) De los otros dos personajes, (3) antagonistas u oponentes, hay (1) identificación (Teseo, Ariadna) pero no caracterización alguna - de hecho, la segunda es solo un nombre, un agente presente pero no actuante-, y sí una (2) intervención actoral del primero (muerte del minotauro) aludida mediante una frase del narrador (la sangre en la espada) y otra intervención del propio personaje (la pregunta a Ariadna).

Dos son las acciones (1) fundamentales, estando la primera, previa y más extensa, subordinada a la segunda, breve, principal y final: la meditación o reflexión de Asterión (relato enmarcado y en primera persona de personaje) y el encuentro de Teseo y Ariadna (relato básico de narrador extra y heterodiegético, cubierto hasta su aparición final). Y tres son las técnicas que integran, construyen y relacionan estas dos acciones básicas: discursivamente, el largo monólogo interior directo o citado; estructuralmente, el suspense, con su alargamiento sintáctico, su modalidad semántica de enigma y la búsqueda intratextual de creciente tensión de cara al lector implícito, incluyendo el reconocimiento o anagnórisis final; lógicamente, la inversión —o quiasmo o flip-flop según Dupriez

\footnotetext{
bien conocidos y de innecesaria y prolija explicación aquí. Así ocurre, en cuanto a la representación funcional de acontecimientos y actores, con los conceptos de motivo de los formalistas rusos y Doležel, de función de Propp y Barthes o las oraciones de Todorov, pero también con los de esfera de acción de Propp, fuerzas dramáticas de Souriau o actantes de Greimas; así ocurre también con nociones (acción en ascenso, expectativa, clímax) vinculadas a la gradación o intensidad narrativa; así ocurre finalmente con la misma lógica de las secuencias simples y complejas citadas por Todorov y sobre todo Bremond.
} 
(Valles, 2002: 418) — de papeles y visiones, al presentar casi todo el relato el narrador heterodiegético con focalización omnisciente desde la perspectiva del minotauro y en primera persona frente a la visión usual, conocida y opuesta del mito clásico, contado desde la perspectiva del héroe/Teseo. La trama está constituida por una serie de acontecimientos (2) — todos ellos ejecutados por Asterión, salvo el final, realizado por Teseo- que se desarrollan autónomamente desde su inicio a fin: a) reflexión inicial sobre sí mismo y la casa, b) salida vespertina al exterior, c) meditación sobre su formación, d) diversos juegos solo y con el amigo imaginario, e) nueva reflexión sobre la casa, f) encuentros con los nueve hombres que entran a la casa cada nueve años, g) meditación sobre su aspecto físico, h) relato de Teseo a Ariadna. La arquitectura de las funciones o motivos (3) básicos de la fábula (enigmas $a$ y $b$-Asterión, casa-, revelaciones progresivas e indirectas respecto a los mismos, descubrimiento o anagnórisis final - minotauro, laberinto-), en la que juegan un papel esencial - muy enlazado con el suspense- las informaciones y sobre todo los indicios en el sentido de Barthes (1974), se relaciona superestructuralmente de un modo claro con la organización secuencial lógica del relato (principio/medio/fin, enigma planteado/enigma desvelándose/enigma resuelto).

La analepsis respecto al tiempo de la intervención del narrador y la pausa reflexiva son la clave constructiva del tiempo (3) del relato, que opera en dos momentos (1) en la fábula: el momento previo a la muerte de Asterión (supuestamente la noche), que encierra sus reflexiones y dura lo que duran sus meditaciones, y el posterior, final y breve, casi instantáneo, a la muerte del minotauro (ya de mañana). Aunque hay más lugares - y objetos- secundarios dentro del laberinto, corresponden a esos dos momentos principales también dos lugares (1) esenciales, que son la casa y el exterior de la misma; la descripción del laberinto, diseminada, indirecta e indicial (galerías, cerraduras, aljibes, corredores...) y marcada por la visión afectiva y poco clara de Asterión, constituye la peana sobre la que se construye el espacio (3) en casi todo el texto, salvo en la indefinición e indeterminación final de la situación de Teseo y Ariadna (exterior del laberinto). La integración del tiempo y el espacio se da en los dos únicos ámbitos de actuación (2) que encierran los nueve acontecimientos citados en el párrafo anterior: el primero, el tiempoespacio de la reflexión mental de Asterión, cubre los siete primeros acontecimientos — de $a$ a $g$-, que se producen dentro de la casa; el segundo, el instante del encuentro entre Teseo y Ariadna, ya de mañana y en el exterior del laberinto, tan solo incluye el último acontecimiento $-h-$.

Tras este breve análisis, realizado a modo de ejemplificación sobre La casa de Asterión de Borges, cabe representar en este cuarto y último cuadro (4) la relación entre los tres estratos y cuatro elementos del texto narrativo: 


\section{CUADRO 4 \\ ESTRATOS Y ELEMENTOS NARRATIVOS}

\begin{tabular}{|c|c|c|c|c|c|}
\hline $\begin{array}{l}\text { ESTRATOS } \\
\text { NARRATIVOS }\end{array}$ & $\begin{array}{c}\text { OBJETO, CARÁCTER } \\
\text { Y ORDENACIÓN }\end{array}$ & \multicolumn{4}{|c|}{ ELEMENTOS NARRATIVOS } \\
\hline $\begin{array}{l}\text { RELATO } \\
\text { plano } \\
\text { enunciativo: } \\
\text { PALABRAS }\end{array}$ & $\begin{array}{l}\text { - El relato (discurso) } \\
\text { - Dimensión verbal } \\
\text { (palabras) } \\
\text { - Orden textual }\end{array}$ & personaje & acción & tiempo & espacio \\
\hline $\begin{array}{l}\text { TRAMA } \\
\text { plano } \\
\text { narrativo: } \\
\text { ACTUACIONES }\end{array}$ & $\begin{array}{l}\text { - Lo relatado (contenido) } \\
\text { - Dimensión escénica } \\
\text { (actuaciones) } \\
\text { - Orden literario }\end{array}$ & actor & acontecimiento & $\begin{array}{r}\text { ámbito } \\
\text { (integración }\end{array}$ & $\begin{array}{l}\text { ctuación } \\
\text { npoespacial }\end{array}$ \\
\hline $\begin{array}{l}\text { FÁBULA } \\
\text { plano lógico: } \\
\text { CATEGORI- } \\
\text { ZACIONES }\end{array}$ & $\begin{array}{l}\text { - Lógica del relato } \\
\text { (macroestructura) } \\
\text { - Dimensión funcional } \\
\text { (funciones) } \\
\text { - Orden cronológico } \\
\text { y causal }\end{array}$ & $\begin{array}{l}\text { actante, } \\
\text { figura }\end{array}$ & $\begin{array}{l}\text { función, } \\
\text { secuencia }\end{array}$ & $\begin{array}{l}\text { momento } \\
\text { (marco } \\
\text { temporal) }\end{array}$ & $\begin{array}{c}\text { lugar } \\
\text { (marco } \\
\text { espacial) }\end{array}$ \\
\hline
\end{tabular}

\section{BIBLIOGRAFÍA CITADA}

Bal, Mieke (1985). Teoría de la narrativa (Una introducción a la narratología). Madrid: Cátedra. Primera ed. de 1977.

Barthes, Roland (1974). «Introducción al análisis estructural de los relatos», en Roland Barthes, A.J. Greimas, Claude Bremond, Jules Gritti, Violette Morin, Christian Metz, Tzventan Todorov y Gérard Genette, Análisis estructural del relato. 3. ${ }^{\mathrm{a}}$ ed. Buenos Aires: Tiempo Contemporáneo, pp. 9-43. Primera ed. de 1966.

Benveniste Emile (1971). Problemas de lingüística general. México D.F.: Siglo XXI. Primera ed. de 1966.

Bobes Naves, María del Carmen (1993). La novela. Madrid: Síntesis.

Booth, Wayne (1974). La retórica de la ficción. Barcelona: Bosch. Primera ed. de 1961.

Borges, Jorge L. (1980). Prosa completa, II. El Aleph. Barcelona: Bruguera. Primera ed. de 1949.

Bourneuf, Roland y Réal Ouellet (1985). La novela. 4. ${ }^{\mathrm{a}}$ ed. Barcelona: Ariel. Primera ed. de 1972.

Bremond, Claude (1973). Logique du récit. Paris: Seuil.

Bremond, Claude (1974). «La lógica de los posibles narrativos», en Roland Barthes, A.J. Greimas, Claude Bremond, Jules Gritti, Violette Morin, Christian Metz, Tzventan Todorov y Gérard Genette, Análisis estructural del relato. 3. ${ }^{\mathrm{a}}$ ed. Buenos Aires: Tiempo Contemporáneo, pp. 87-110. Primera ed. de 1966.

Brooks, Peter (1984). Reading for the Plot. Deisgn and Intention in Narrative. Oxford: Clarendon Press.

Chatman, Seymour (1990). Historia y discurso. La estructura narrativa en la novela y en el cine. Madrid: Taurus. Primera ed. de 1978. 
Chico Rico, Francisco (1988). Pragmática y construcción literaria. Discurso retórico y discurso narrativo. Alicante: Universidad de Alicante.

Cohn, Dorrit (1981). La transparence intérieur. Modes de representation dans la vie psychique dans le roman. Paris: Seuil. Primera ed. Transparent Minds, 1978.

Cuddon, John Anthony (ed.) (1998). A Dictionary of Literary Terms and Literary Theory. 4. ${ }^{\text {a }}$ ed. Oxford: Blackwell Publishers. Primera ed. de 1976.

Culler, Jonathan (1978). La poética estructuralista. El estructuralismo, la lingüística y el estudio de la literatura. Barcelona: Anagrama. Primera ed. de 1975.

Dijk, Teun A. Van (1972). Some Aspects of Text Grammar. The Hague: Mouton.

Dijk, Teun A. Van (1974-1975). «Action, Action Description and Narrative», New Literary History. V/I, pp. 273-294.

Dijk, Teun A. Van (1980). Texto y contexto. Madrid: Cátedra. Primera ed. de 1977.

Doležel, Lubomír (1972). «From Motifemes to Motifs». Poetics, 4, pp. 55-90

Doležel, Lubomír (1986). «Semiotics of Literary Communication». Strumenti Critici, 1, pp. $5-48$.

Domínguez Caparrós, José (2002). Teoría de la literatura. Madrid: Centro de Estudios Ramón Areces.

Erlich, Viktor (1974). El formalismo ruso. Barcelona: Seix Barral. Primera ed. 1955.

Fludernik, Monika (1996). Towards a «Natural» Narratology. London: Routledge.

Forster, Edward M. (1983). Aspectos de la novela. Madrid: Debate. Primera ed. 1927.

García Barrientos, José Luis (1991). Drama y tiempo: Dramatología I. Madrid: Consejo Superior de Investigaciones Científicas.

García Landa, José Ángel (2005). Narrative Theory [en línea]. Zaragoza: Universidad de Zaragoza, disponible en < http://www.unizar.es/departamentos/filologia_inglesa/garciala/ publicaciones/narrativetheory/0.Introduction.htm> [ref. de 26/7/2016]. Primera ed. 1990.

Garrido Domínguez, Antonio (1993). El texto narrativo. Madrid: Síntesis.

Garrido Domínguez, Antonio (2011). Narración y ficción. Literatura e invención de mundos. Madrid - Frankfurt: Iberoamericana - Vervuert.

Genette, Gérard (1972). Figures, III. Paris: Seuil.

Genette, Gérard (1998). Nuevo discurso del relato. Madrid: Cátedra. Primera ed. 1993.

Gnutzmann, Rita (1994). Teoría de la literatura alemana. Madrid: Síntesis.

Greimas, Algirdas J. y J. Courtès (1991). Semiótica. Diccionario razonado de la teoría del lenguaje, II. Madrid: Gredos. Primera ed. 1979.

Hawthorn, Jeremy (1997). Studying the novel. An Introduction. 3. a ed. London: Arnold. Primera ed. 1985.

Hawthorn, Jeremy (2000). A Glossary of Contemporary Literary Theory. London: Arnold.

Herman, David (ed.) (2003). Narrative Theory and the Cognitive Sciences. Stanford: CSLI Publications.

Núñez Ramos, Rafael (2010). El pensamiento narrativo. Aspectos cognitivos del relato. Oviedo: Universidad de Oviedo.

Onega, Susana y José Ángel García Landa (ed.) (1996). Narratology. Harlow: Longman.

Preminger, Alex y T.V.F. Brogan (ed.) (1993). The New Princeton Encyclopedia of Poetry and Poetics. Princeton: Princeton University Press.

Prince, Gerald (1987). A Dictionary of Narratology. Lincoln \& London: University of Nebraska Press.

Ricoeur, Paul (1980). «Narrative Time», Critical Inquiry. 7, 1, pp. 169-190.

Rimmon-Kenan, Shlomith (1983). Narrative Friction: Contemporary Poetics. London: Methuen.

Rodríguez Pequeño, Mercedes (1995). Teoría de la literatura eslava. Madrid: Síntesis.

Scholes, Robert (1981). Introducción al estructuralismo en literatura. Madrid: Gredos. Primera ed. de 1974. 
Segre, Cesare (1976). Las estructuras y el tiempo. Barcelona: Planeta. Primera ed. 1974. Segre, Cesare (1985). Principios de análisis del texto literario. Barcelona: Crítica.

Shklovski, Viktor V. (1975). Una teoria della prosa. Torino: Einaudi. Primera ed. de 1925. Stanzel, Franz K. (1988). A Theory of Narrative. Cambridge: Cambridge University Press. Primera ed. 1982.

Sternberg, Meir (2003a). «Universals of Narrative and Their Cognitivist Fortunes (I)», Poetics Today. 24, 2, pp. 297-395.

Sternberg, Meir (2003b). «Universals of Narrative and Their Cognitivist Fortunes (II)», Poetics Today. 24, 3, pp. 517-638

Sullà, Enric (ed.) (1996). Teoría de la novela. Antología de textos del siglo XX. Barcelona: Crítica.

Tacca, Óscar (1978). Las voces de la novela. 2. ${ }^{a}$ ed. Madrid: Gredos. Primera ed. 1973.

Todorov, Tzvetan (1973). Poétique. Paris: Seuil. Primera ed. 1968.

Todorov, Tzvetan (1974) «Las categorías del relato literario», en Roland Barthes, A.J. Greimas, Claude Bremond, Jules Gritti, Violette Morin, Christian Metz, Tzventan Todorov y Gérard Genette, Análisis estructural del relato. 3. ${ }^{\mathrm{a}}$ ed. Buenos Aires: Tiempo Contemporáneo, pp. 155-192. Primera ed. 1966.

Tomashevski, Boris (1982). Teoría de la literatura. Madrid: Akal. Primera ed. 1928.

Valles Calatrava, José R. (1999). El espacio en la novela. Organización y funcionamiento del espacio narrativo en «La ciudad de los prodigios» de Eduardo Mendoza. Almería: G.I. de Teoría de la Literatura y Literatura Comparada.

Valles Calatrava, José R. (dir.) (2002). Diccionario de teoría de la narrativa. Granada: Alhulia.

Valles Calatrava, José R. (2008). Teoría de la narrativa. Una perspectiva sistemática. Madrid - Frankfurt: Iberoamericana - Vervuert.

Villanueva, Darío (1989). El comentario de textos narrativos: la novela. Gijón: Júcar-Aceña.

Fecha de recepción: 27 de abril de 2015.

Fecha de aceptación: 24 de julio de 2015. 